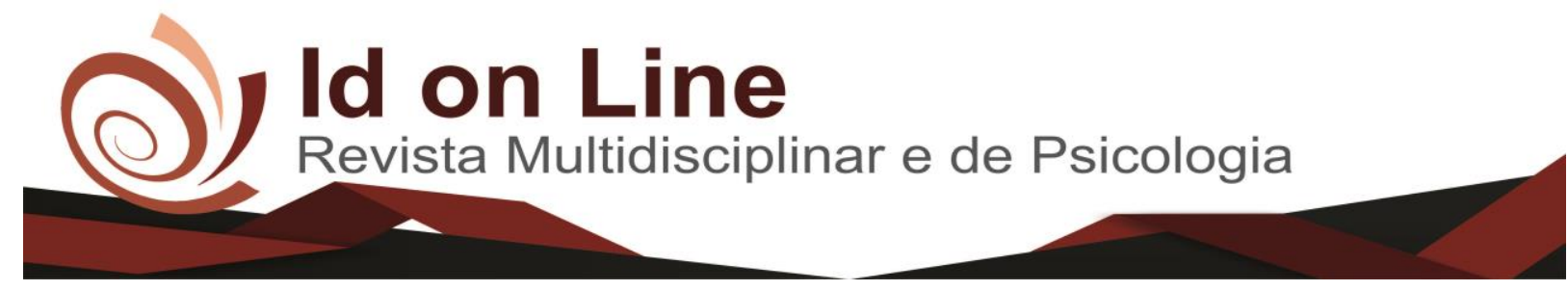

Artigo

\title{
Qualidade de vida e percepção corporal com o tratamento de radiofrequência na adiposidade abdominal
}

Beatriz Souza Santos ${ }^{1}$; Karla Cavalcante Silva de Morais² ; Jessica Almeida Barros ${ }^{3}$; Susan Caroline Santos Ferreira ${ }^{4}$; Thais Rocha da Silva ${ }^{5}$; Juliana Barros Ferreira ${ }^{6}$;

\begin{abstract}
Resumo: Este estudo tem como objetivo analisar a influência da percepção corporal na qualidade de vida de mulheres submetidas ao tratamento com a radiofrequência na adiposidade abdominal. Trata-se de um estudo experimental de delineamento transversal, analítico com abordagem quantitativa, realizado em onze mulheres de 18 a 25 anos com queixa de adiposidade abdominal, em um núcleo de práticas em fisioterapia de uma intuição privada na cidade de Vitória da Conquista/Bahia. Foram aplicados um questionário sociodemográfico, o questionário de qualidade de vida Whoqol-Bref e a Escala de Silhuetas, além da avaliação da adipometria e perimetria abdominal antes e após o tratamento com a radiofrequência. Todas as participantes receberam o tratamento por meio da radiofrequência, em região abdominal, a uma temperatura de $36^{\circ} \mathrm{a} 38^{\circ} \mathrm{C}$, por um período de dois meses, totalizando dez sessões. Após as 10 sessões, foi possível constatar que a radiofrequência foi eficaz no tratamento da adiposidade abdominal, reduzindo a adipometria e perimetria abdominal, e promove uma melhora da qualidade de vida e percepção corporal.
\end{abstract}

Palavras-chave: Imagem Corporal. Percepção. Qualidade de Vida. Radiofrequência.

\section{Quality of life and body perception with radiofrequency treatment in abdominal adiposity}

\begin{abstract}
This study aims to analyze the influence of body perception on the quality of life of women submitted to treatment with radiofrequency in abdominal adiposity. This is an experimental, cross-sectional, analytical study with a quantitative approach, performed in eleven women aged 18 to 25 years with abdominal adiposity complaint, in a nucleus of physiotherapy practices of a private intuition in the city of Vitória da Conquista / Bahia . A sociodemographic questionnaire was used, the Whoqol-Bref quality of life questionnaire and the Silhouettes Scale, as well as the evaluation of adipometry and abdominal perimetry before and after treatment with radiofrequency. All participants were treated by radiofrequency, in the abdominal region, at a temperature of $36^{\circ}$ to $38^{\circ} \mathrm{C}$, for a period of two months, totaling ten sessions. After 10 sessions, it was possible to verify that radiofrequency was effective in the treatment of abdominal adiposity, reducing adipometry and abdominal perimetry, and promoting an improvement in the quality of life and body perception.
\end{abstract}

Keywords: Body Image. Perception. Quality of life. Radiofrequency.

\footnotetext{
1,3,4 Graduanda em Fisioterapia, Faculdade Independente do Nordeste-FAINOR, http://www.fainor.com.br, Vitória da Conquista, Bahia, Brasil.

${ }^{2}$ Fisioterapeuta, Mestre em Saúde Pública, Docente da Faculdade Independente do Nordeste-FAINOR, http://www.fainor.com.br, Docente da Faculdade Maurício de Nassau, https://www.uninassau.edu.br/institucional/nacional, Vitória da Conquista, Bahia, Brasil.

${ }^{5}$ Tecnóloga em Estética e Cosmética, Faculdade Independente do Nordeste-FAINOR, http://www.fainor.com.br, Vitória da Conquista, Bahia, Brasil.

${ }^{6}$ Fisioterapeuta, Mestre em Tecnologias em Saúde, Docente da Faculdade Independente do Nordeste-FAINOR, http://www.fainor.com.br, Vitória da Conquista, Bahia, Brasil.

*Autor correspondente: Beatriz Souza Santos, e-mail: bekao10@hotmail.com|. Telefone: (77) 9.8853-6120
} 


\section{Introdução}

A percepção corporal está diretamente relacionada à autoimagem, onde o indivíduo faz uma assimilação de si mesmo, envolvendo questões sentimentais, psicológicas e sociais. Esses aspectos influenciam de diversas formas na qualidade de vida e autoestima pessoal (CASTILHO, 2001). Fato esse, comprovado em estudos realizados com pessoas submetidas a diversos procedimentos estéticos corporais (SECCHI; CAMARGO; BERTOLDO, 2009).

Aguiar (2006) afirma que diante da necessidade de se ter um corpo perfeito, aquele imposto pela sociedade e pelas redes sociais, as pessoas estão sendo movidas a uma busca incessante por uma boa aparência física. E esta busca em satisfazer os desejos estéticos também está associada ao mercado da beleza, o qual utiliza-se de várias inovações tecnológicas (MENDONÇA, 2005). No Brasil, foram realizados mais de 2,5 milhões de procedimentos estéticos, sendo cirúrgicos ou não, perdendo apenas para os Estados Unidos, que registrou cerca de três milhões de procedimentos (JANSEN ,2010).

Segundo Costa e Meyer (2008), uma das disfunções estéticas que mais está incomodando os indivíduos é a adiposidade abdominal. Com isso, surgem cada vez mais tratamentos que prometem corrigir tal disfunção, sobretudo para o gênero feminino (FREITAS et al., 2010). Para tratar a adiposidade abdominal, existem procedimentos que atuam de forma invasiva e não invasiva (DUARTE, MEJIA, 2014). Dentre os recursos não invasivos, destacase a radiofrequência (RF) que é utilizada para redução de celulite, flacidez, gordura localizada, rejuvenescimento facial, entre outros (SILVA et al., 2014).

A Radiofrequência (RF) foi desenvolvida pelo fisiologista francês Jaques Arsène D' Arsonval, é um recurso que consiste na aplicação por meio de uma técnica que gera calor logo abaixo da pele por meio de ondas com frequência de 1,2 $\mathrm{MHz}$ e 2,4 MHz e potência de 100W. (LOFEU et al., 2015; DUARTE, MEJIA, 2014).

A RF funciona a partir de dois principais mecanismos de ação, vasodilatação e aquecimento tecidual, que atuam através de um campo elétrico oscilante que gera calor através de colisão de moléculas de água (FRANCO, KOTHARE, GOLDBERG, 2009). O campo elétrico se desenvolve e é direcionado de forma perpendicular para a interface subcutânea da pele, atingindo o tecido adiposo atuando de forma seletiva, evoluindo para um alto nível elétrico de impedância e condutividade em relação à derme gerando ruptura de adipócitos produzindo 
uma queima de gordura localizada (FRANCO et al, 2010; FELIPE, REDONDO, 2007; KAPLAN, GAT, 2009).

Diante da crescente procura por tratamentos estéticos, estudos que comprovem técnicas de tratamento benéficas utilizadas pela fisioterapia dermatofuncional, que sejam não invasivas de baixo custo e eficazes na redução da adiposidade abdominal que avaliem a percepção corporal e a qualidade de vida são de extrema necessidade e importância. Uma vez que irá contribuir na expansão do campo científico sobre a temática em questão e no aperfeiçoamento e melhoria das técnicas e ainda aumentar a satisfação e qualidade de vida das pessoas que se submetem a esses procedimentos (TOMASSO, 2008).

Este estudo teve como objetivo avaliar a eficácia da aplicação da técnica da RF, e comparar a qualidade de vida e percepção corporal antes e após o tratamento com a radiofrequência.

\section{Materiais e Métodos}

Trata-se de um estudo experimental, de delineamento transversal, analítico e com análise quantitativa, realizado em um Núcleo de Práticas em Fisioterapia (NEF), localizada na cidade de Vitória da Conquista - BA. Considerada a terceira maior cidade do Estado da Bahia, localizada a $503 \mathrm{Km}$ da capital Salvador, com população de, aproximadamente, 336.987 habitantes e compreendendo, aproximadamente, oitenta municípios na Bahia e dezesseis no norte de Minas Gerais (IBGE, 2010).

Todas as etapas da pesquisa seguiram rigorosamente os princípios éticos da resolução 466/12 do Conselho Nacional de Saúde. Todas os participantes assinaram um Termo de Consentimento Livre e Esclarecido (TCLE), comprovando sua participação voluntária nesta pesquisa. Este estudo foi submetido ao Comitê de Ética e Pesquisa (CEP) a Faculdade Independente do Nordeste (FAINOR), conforme protocolo CAAE: 68339517.9.0000.5578 e número do parecer de aprovação: 2.108.559.

O estudo foi realizado a partir de uma amostra por conveniência que foi composta por estudantes do curso de fisioterapia, com idade entre 18 a 25 anos e limitou-se exclusivamente com participantes do sexo feminino. Tendo como critérios de inclusão, mulheres com adiposidade abdominal praticantes de atividade física. Foram exclusas da pesquisa mulheres 
que tinham patologias hematológicas, que façam uso de dispositivo intrauterino (DIU), apresentem doenças crônicas degenerativas e neurológicas.

As informações como raça, renda e estado civil foram obtidas através do questionário sociodemográfico. A qualidade de vida foi analisada pelo questionário Whoqol-Bref que é composto por 26 questões, sendo definida por 4 domínios: físico, psicológico, relações sociais e meio ambiente, onde o grau de satisfação é avaliado com escala oferecida de cinco pontos, que classificou o nível em: 1 - muito insatisfeita; 2 - insatisfeito; 3 - pouco satisfeito; 4 satisfeito; 5 - muito satisfeito. (FLECK, 2000; PEREIRA, TEIXEIRA, ANDRADE, LOPES, 2014).

A escala de silhuetas de Stunkard foi utilizada para avaliação da imagem corporal, sendo que as imagens das escalas geralmente variam de um sujeito muito magro a um obeso. As figuras representaram a atual imagem do indivíduo e como o mesmo deseja ficar. A insatisfação corporal é representada pela discrepância entre essas medidas. Contem nove figuras para cada sexo. Para esta pesquisa foi utilizada somente as figuras referentes ao sexo feminino (CORTÊS et al, 2013).

Após preencher os questionários, as participantes foram submetidas a avaliação da adipometria abdominal, realizada com adipômetro científico da marca Sanny®, que foi posicionado na prega vertical à direita da cicatriz umbilical com distância de $2,5 \mathrm{~cm}$ da região supracitada (BORGES, 2016). Em sequência foi realizado uma avalição perimétrica na região abdominal, realizada com a fita métrica marca ISP®, a mesma foi posicionada a $2,5 \mathrm{~cm}$ acima e abaixo da cicatriz supra e infraumbilical (BORGES, 2016).

O aparelho de RF utilizado foi da marca HTM®, com intensidade inicial e final de $50 \%$ e frequência de $1.2 \mathrm{MHz}$, com a manopla corporal concêntrica de transferência capacitiva. A técnica de aplicação foi realizada em uma sala reservada. A marcação da região abdominal foi realizada em quadrantes (AGNE, 2011) e posteriormente foi aplicado o gel glicerinado manipulado para transferência da RF. As participantes foram posicionadas em decúbito dorsal na maca de marca ISP (Instituto São Paulo) para condução da RF. A temperatura era mensurada simultaneamente à movimentação circular do eletrodo com um termômetro infravermelho até atingir a temperatura de $36^{\circ} \mathrm{C}$ a $38^{\circ} \mathrm{C}$. Após atingir a temperatura o movimento circular foi mantido na área demarcada por 2 minutos. A participante foi orientada a não utilizar nenhum tipo de loção hidratante ou óleo corporal antes das sessões. 
As aplicações de RF foram realizadas nos turnos matutino e vespertino, duas vezes por semana, durante dois meses por uma graduanda de Fisioterapia acompanhada pela pesquisadora responsável. Cada participante foi atendida por 10 sessões, e o tempo de tratamento teve duração de 20 minutos.

A apreciação estatística desta pesquisa foi realizada pelo pacote estatístico SPSS (Statistical Package for Social Sciences) versão 20.0. Os resultados estão apresentados por meio de tabelas e figuras; as variáveis categóricas, expressas em valores absolutos e percentuais $-\mathrm{n}$ (\%). Para a análise da comparação foi utilizado o Test de T Student.

\section{Resultados}

Foram analisados, de acordo critérios de inclusão da pesquisa, questionários e dados referentes a 11 mulheres, que foram aplicados antes e após as 10 sessões de Radiofrequência de transferência não ablativa na adiposidade abdominal.

Conforme pode ser visto na Tabela 1, sobre o questionário sociodemográfico, no qual pelo critério de seleção da amostra, as variáveis idade e escolaridade foram excluídas. A maior parcela das mulheres participantes do estudo se identificaram como solteiras, com percentual de $90,9 \%$. Quanto a raça, 54,5\% das pesquisadas se declararam pardas, enquanto apenas $9,1 \%$ se manifestou como negra. Em relação ao questionamento sobre renda familiar, 81,8\% respondeu não obter renda ou não saber.

Tabela 1. Características sociodemográficas da amostra. Vitória da Conquista - BA, 2017.

\begin{tabular}{lccc}
\hline Variáveis & \% respostas & n & \% \\
\hline Estado civil & 100 & & \\
$\quad$ Solteira & & 10 & 90,9 \\
$\quad$ Casada & 100 & 1 & 9,1 \\
Raça & & 4 & 36,4 \\
$\quad$ Branca & & 1 & 9,1 \\
$\quad$ Negra & 100 & 6 & 54,5 \\
$\quad$ Parda & & & 81,8 \\
Renda familiar & & 9 & 18,2 \\
$\quad$ Sem renda/não sabe & & 2 & \\
$\quad$ Até um salário mínimo
\end{tabular}

Fonte: Dados da pesquisa; $\%=$ percentual; $\mathrm{n}=$ número de participantes. 
A Tabela 2 contém informações sobre os dados de perimetria e adipometria abdominal, na qual foi identificado na perimetria supraumbilical uma diferença média de 2,64 cm no antes e depois da RF, tendo média de $81,00 \mathrm{~cm}$ antes das sessões e após 78,36 cm com p =0,005. No que refere-se a perimetria infraumbilical notou-se uma média de 91,45 pré e 87,36 pós RF, apresentando redução de 4,09 cm e com significância de 0,0004. Com relação a adipometria abdominal em borda lateral notou-se uma média de 22,96 e 20,00 antes e depois respectivamente das sessões, e apresentando $\mathrm{p}=0,0027$.

Tabela 2. Medidas de perimetria e adipometria antes e depois da RF. Vitória da Conquista - BA, 2017.

\begin{tabular}{|c|c|c|c|c|c|c|}
\hline Variáveis & An & & Dep & $\mathbf{R F}$ & & \\
\hline & Média & $\mathrm{DP}^{1}$ & Média & DP & Df-m² & Sig. ${ }^{3}$ \\
\hline Perimetria & & & & & & \\
\hline Supraumbilical & 81,00 & $\pm 7,33$ & 78,36 & $\pm 6,69$ & 2,64 & 0,005 \\
\hline Infraumbilical & 91,45 & $\pm 7,43$ & 87,36 & $\pm 6,24$ & 4,09 & 0,0004 \\
\hline Adipometria & & & & & & \\
\hline Borda lateral $^{4}$ & 22,96 & $\pm 8,63$ & 20,00 & $\pm 7,37$ & 2,96 & 0,0027 \\
\hline
\end{tabular}

A tabela 3 ressalta dados obtidos através do questionário Whoqol-Bref, que é responsável por analisar qualidade de vida, e que foram aplicados antes e depois das aplicações de RF. Houve melhora no que refere-se a satisfação com a saúde, que antes apresentava uma média de 3,82 para 3,91 $( \pm 0,75$ e 0,94$)$ respectivamente, apresentando significância de $p=$ 0,002. O outro ponto na qual obteve-se uma melhora após a RF foi no domínio meio ambiente no qual, antes 8 mulheres indicaram como regular e 3 como necessidade de melhorar e depois 9 indicaram como regular e 2 como necessidade de melhorar, obtendo um $\mathrm{p}=0,048$.

Tabela 3. Qualidade de vida WHOQOL-bref antes e depois RF. Vitória da Conquista BA, 2017.

\begin{tabular}{lccccc}
\hline Variáveis & \multicolumn{2}{c}{ Antes } & \multicolumn{3}{c}{ Depois } \\
\hline & Média & DP & Média & DP & sig $^{\mathbf{2}}$ \\
\cline { 2 - 6 } $\begin{array}{l}\text { Qualidade de vida geral } \\
\begin{array}{l}\text { Percepção da } \\
\text { qualidade de vida }\end{array}\end{array}$ & 3,91 & 0,70 & 3,91 & 0,70 & 0,003 \\
$\begin{array}{l}\text { Satisfação com a } \\
\text { saúde }\end{array}$ & 3,82 & 0,75 & 3,91 & 0,94 & 0,002
\end{tabular}




\begin{tabular}{|c|c|c|c|c|c|c|}
\hline \multirow{3}{*}{$\begin{array}{l}\text { Domínio físico } \\
\text { Regular }\end{array}$} & \multirow{3}{*}{100} & & & & & \multirow{3}{*}{0,25} \\
\hline & & & & & & \\
\hline & & 11 & 100 & 11 & 100 & \\
\hline Domínio psicológico & \multirow[t]{3}{*}{100} & & & & & \\
\hline Necessita melhorar & & 2 & 18,2 & 2 & 18,2 & \\
\hline Regular & & 9 & 81,8 & 9 & 81,8 & 0,78 \\
\hline $\begin{array}{l}\text { Domínio relações } \\
\text { sociais }\end{array}$ & \multirow[t]{3}{*}{100} & & & & & \multirow{3}{*}{0,72} \\
\hline Necessita melhorar & & 1 & 9,1 & 1 & 9,1 & \\
\hline Regular & & 10 & 90,9 & 10 & 90,9 & \\
\hline Meio ambiente & \multirow[t]{3}{*}{100} & & & & & \multirow{3}{*}{0,048} \\
\hline Necessita melhorar & & 3 & 27,3 & 2 & 18,2 & \\
\hline Regular & & 8 & 72,7 & 9 & 81,8 & \\
\hline
\end{tabular}

${ }^{1}$ Desvio Padrão; ${ }^{2}$ Significância pelo teste t-Student. \% = percentual; $\mathrm{n}=$ número de indivíduos; Fonte: Dados da pesquisa.

A tabela 4 condensa a percepção do corpo antes e depois do tratamento da RF, indicando uma melhora da percepção corporal, principalmente com uma predominância no que antes era da figura 5, com 4 indivíduos $(36,4 \%)$, e passou a ser na figura 4 com 4 indivíduos $(36,4 \%)$. Outro dado interessante é que a imagem da figura 7 que apareceu 2 vezes antes, deixou de aparecer depois da RF.

Tabela 4. Percepção corporal antes e depois da RF. Vitória da Conquista - BA, 2017.

\begin{tabular}{lcccc}
\hline Silhuetas & \multicolumn{2}{c}{ ANTES } & \multicolumn{2}{c}{ DEPOIS } \\
\hline Imagens & $\mathbf{n}$ & \% & n & \% \\
Figura 2 & 1 & 9,1 & 2 & 18,2 \\
Figura 3 & 2 & 18,2 & 3 & 27,3 \\
Figura 4 & 1 & 9,1 & 4 & 36,4 \\
Figura 5 & 4 & 36,4 & 1 & 9,1 \\
Figura 6 & 1 & 9,1 & 1 & 9,1 \\
Figura 7 & 2 & 18,2 & & \\
\hline
\end{tabular}

Fonte: Dados da pesquisa. $\%=$ percentual; $\mathrm{n}=$ número de indivíduos

\section{Discussão}

O questionário sociodemográfico verificou que a maioria das mulheres que realizaram tratamento com a radiofrequência em região abdominal, em relação ao estado civil se declaram 
solteira, o que corrobora com o estudo de Ferreira, Silva e Lemos (2016), que indica uma crescente preocupação estética das mulheres solteiras. Dentro da autodenominação racial, houve uma maior frequência de pardas, o que é condizente com os dados do IBGE de 2010, que indica que a maior parte da população brasileira se auto caracteriza parda.

A pesquisa realizada por Paxton, et al em 2006, demonstra que as mulheres de etnia brancas e pardas apresentaram maior insatisfação com o seu corpo, tendo uma maior tendência a fazer tratamentos estéticos afim de estabelecerem padrões de beleza impostos pela mídia.

Dentre as medidas de adiposidade abdominal por meio de adipometria e perimetria apresentadas através de uma comparação do antes e depois das aplicações de radiofrequência, houve uma redução em todas as medidas realizadas nas participantes do estudo. No estudo de Tommaso et al em 2016, indica que a radiofrequência tem efeitos positivos na redução da adiposidade abdominal em relação a adipometria.

Esta redução da adipometria e da perimetria podem ser justificadas pela ação da radiofrequência na produção de energia que atravessa as células epiteliais, chegando a atingir células musculares que, por sua vez, produzem uma rápida fricção provocando um aumento da temperatura tissular (MAYORAL, 2007; HEXSEL,2011). Esta elevação da temperatura acarreta uma vasodilatação que melhora o trofismo tissular, gerando uma maior reabsorção de líquidos intersticiais, melhorando a drenagem de resíduos celulares, o que contribui para uma redução em medidas abdominais. (GÓMEZ, 2007).

O estudo realizado por Manuskiatti et al (2009), indica que após o tratamento realizado com 3 sessões semanais por 4 semanas, em 39 mulheres, 37 dessas mulheres apresentaram uma redução de média de 3,50 cm de perimetria abdominal supraumbilical e 4,61 infraumbilical, indicando $\mathrm{p}=0,002$ de significância. $\mathrm{O}$ que corrobora com esta pesquisa que indica uma redução de média de 2,64 cm na região supraumbilical e 4,09 com de infraumbilical, com significância de $\mathrm{p}=0,005$ e $\mathrm{p}=0,0004$ respectivamente.

A qualidade de vida relaciona-se com fatores ligados à vida profissional, acadêmica, familiar, religiosa e todas as outras a qual faz parte do dia-a-dia, inclusive a autoestima, sendo também uma questão importante para relacionar com imagem corporal (PEREIRA et al., 2014). A partir do senso comum, a alteração na qualidade de vida corresponde a estudos que indicam que a mesma é uma união das variáveis explicando assim sua melhora (HALLAWELL, 2008).

De acordo aos resultados encontrados neste estudo sobre a percepção do corpo de antes e depois do tratamento da RF, houve uma melhora da percepção corporal, em concordância 
com os resultados do estudo realizado por SOAIGHER, ACENCIO e CORTEZ em 2016, mostra que a percepção corporal está diretamente relacionada à autoimagem. Esta autoimagem tem associação como o indivíduo tem uma percepção sobre si em relação aos sentimentos, pensamentos e relações (WARREN, 2005).

Esses aspectos influenciam de diversas formas na qualidade de vida e autoestima do indivíduo, onde a percepção é notória principalmente em relação ao corpo e suas necessidades, sendo comumente analisado em indivíduos submetidos a diversos procedimentos estéticos corporais (CAMPOS, RODRIGUES NETO, 2008).

Limitação: Registro fotográfico para comparar os resultados e como perspectiva a realização de um estudo com uma população maior e um acompanhamento dos resultados por um tempo maior.

\section{Conclusão}

O presente estudo constatou que o tratamento da Radiofrequência é eficaz e reduz a adiposidade e perimetria abdominal, e tem influência positiva na qualidade de vida e na percepção corporal das mulheres. Diante do exposto, torna-se importante a realização de novos estudos, com uma maior amostra populacional, para contribuir cientificamente com a temática em questão.

\section{Referências}

AGNES, J. E. Eu sei eletroterapia. $2^{\mathrm{a}}$ ed. Santa Maria: Sociedade Vicente Pallotti, 2011. 226$253 \mathrm{p}$.

AGUIAR, T. Personal Stalyst: Guia para Consultores de imagem. 4a ed. São Paulo: Senac, 2006.

BORGES, F. S.; SCORZA, F.A. Terapêutica em estética: conceitos e técnicas. São Paulo: Phorte; 2016.

CAMPOS, M. O.; RODRIGUES NETO, J. F. Qualidade de 409 Revista Pesquisa em Fisioterapia. 2016 Nov;6(4):402-410 vida: um instrumento para promoção de saúde. Revista Baiana de saúde pública. 2008;32(2):232- 240. 
CASTILHO, S. M. A imagem corporal. Santo André: ESETec; 2001.

CORTÊS, M. G. et al, $O$ uso de escalas de silhuetas na avaliação da satisfação corporal de adolescentes: revisão sistemática da literatura. Caderno de Saúde Pública, Rio de Janeiro, v. 29, n. 3. p:427-444, mar, 2013.

COSTA, E. M.; MEYER P. F. Avaliação dos Efeitos do uso da Tecaterapia na Adiposidade Abdominal. Trabalho de conclusão de curso, Universidade Potiguar, curso de fisioterapia. Natal. 2008.

DUARTE, A. B.; MEJIA, D. P. M. A utilização da Radiofrequência como técnica de tratamento da flacidez corporal. Portalbio; v.1, n.2 p 77-82, 2014.

FELIPE I, REDONDO P. Animal model to explain fat atrophy using nonablative radiofrequency. Dermatol Surg, v.5, ed, 33, p.141-5, 2007.

FERREIRA, J. B.; SILVA, T. R.; LEMOS, L. M. A. Qualidade de vida, imagem corporal e satisfação nos tratamentos estéticos. Revista Pesquisa em Fisioterapia, v. 6, n.4, p. 402-410, 2016.

FLECK, M. P. A. et. al. Aplicação da versão em português do instrumento abreviado de avaliação da qualidade de vida "WHOQOL-bref”. Revista de saúde pública, n.34, v.2, p. 178$183,2000$.

FRANCO, W.; KOTHARE, A.; GOLDBERG, D. J. Controlled volumetric heating of subcutaneous adipose tissue using a novel radiofrequency technology. Lasers Surg Med 2009;

FRANCO, W.; et. al. Hyperthermic injury to adipocyte cells by selective heating of subcutaneous fat with a novel radiofrequency device: feasibility studies. Lasers Surg Med, 2010.

FREITAS, C. M. S. M. et. al. O Padrão de Beleza Corporal Sobre o Corpo Feminino Mediante $o I M C$. Revista brasileira de Educação Física e Esporte, São Paulo, v.24, n.3, p.389-404, jul./set. 2010

GOMÉZ, A. C. Radiofrequência capacitiva em Celulitis. Casuística. Anais do XVI Congresso Mundial de Medicina Estética. Argentina: Buenos Aires; 2007.

HALLAWELL, P. Visagismo: harmônia e estética. 4a ed. São Paulo:Senac, 2008.

HEXSEL, D. M. et al. A bipolar radiofrequency, infrared, vacuum and mechanical massage device for treatment of cellulite: a pilot study. Journal of Cosmetic and Laser Therapy, v. 13, $\mathrm{n}$. 6, p. 297-302, 2011.

IBGE. Brasil tem mais mulheres. 2010. Disponível em: <http://7a12.ibge.gov.br/vocesabia/ curiosidades/brasil-tem-mais-mulheres.html >. Acesso em: 12 de março de 2017. 
JANSEN R. Pela cirurgia plástica, brasileiras buscam seios e nádegas maiores [Internet]. 2010 [Acessado em 29 de setembro de 2017]. Disponível em: http://oglobo.globo.com/vivermelhor/ mulher/mat/2010/08/15/pela-cirurgiaplastica-brasileiras-buscam-seios-nadegasmaiores-

917398162.asp

KAPLAN, H.; GAT, A. Clinical and histopathological results following TriPollar radiofrequency skin treatments. J Cosmet Laser Ther 2009.

LOFEU, G. M. et. al. Atuação da Radiofrequência na Gordura Localizada no Abdômen: revisão de literatura. Revista da Universidade Vale do Rio Verde, Três Corações, v. 13, n. 1, p. 571-581, 2015.

MANUSKIATTI, W. et. al. TriPollar - Aparelho de radiofrequência para redução do volume abdominal e tratamento da celulite: Estudo piloto. Rev. The Authors: Academina Europeia de Dermatologia e Venerologia, 2009. DOI: 10.1111/j.1468-3083.2009.03254.x

MAYORAL, F. A. Skin tightening with a combined unipolar and bipolar radiofrequency device. J. Drugs. Dermatol. 2007 Feb 6(2):212-5.

MENDONÇA, N. O. Saúde, obesidade e indústria cultural: implicações para o surgimento da indústria do emagrecimento. Monografia (Graduação em Educação Física) - Eseffego, Goiânia, 2005.

PAXTON, S. J.; et. al. Body dissatisfaction prospectively predicts depressive mood and low self-esteem in adolescent girls and boys. J Clin Child Adolesc Psychol. 2006;35(4):539-49. doi: 10.1207/s15374424jccp3504_5.

PEREIRA et al. O trabalho docente e a qualidade de vida dos professores na educação básica. Rev. salud pública. 16, 221-231, 2014.

PEREIRA EF, TEIXEIRA CS, ANDRADE RD, LOPES AS. O trabalho docente e a qualidade de vida dos professores na educação básica. Rev. salud pública, v.16, ed. 2, p. 221-231,2014.

SECCHI, K.; CAMARGO, B. V.; BERTOLDO, R. B. Percepção da Imagem Corporal e Representações Sociais do Corpo. Psicologia: Teoria e Pesquisa Abr-Jun, Vol. 25 n. 2, pp. 229236, 2009.

SILVA, A. R.; et al. Radiofrequência no Tratamento das Rugas Faciais. Revista da Universidade Ibirapuera - São Paulo, v. 7, p. 38-42, jan/jun. 2014.

SOAIGHER, K. A.; ACENCIO, F. R.; CORTEZ, D. A. G. O poder da vaidade e do autocuidado na qualidade de vida. Revista Cinergis, Santa Cruz do Sul, 18, 452-455, jan./mar. 2016. 
TOMMASO, M. A. et. al. Autoimagem e a interface beleza - doença. 2016. Disponível em: $<$ http://tommaso. psc.br/autoimagem-e-a-interface-beleza-doenca/ >. Acesso em 20 de agosto de 2017.

TOMMASO, M. A. Beleza feminina, Saúde e Autoestima. São Paulo, 2008.

WARREN, C. et. al. Ethnicity as a protective factor against internalization of a thin ideal and body issatisfaction. International Journal of Eating Disorders, v.37, p. 241-249, 2005.

\section{Como citar este artigo (Formato ABNT):}

SANTOS, Beatriz S.; MORAIS, Karla C. S de; BARROS, Jessica A.; FERREIRA, Susan C. S.; SILVA, Thais R. da; FERREIRA, Juliana B. Qualidade de vida e percepção corporal com o tratamento de radiofrequência na adiposidade abdominal. Id on Line Revista Multidisciplinar e de Psicologia, 2017, vol.11, n.38, p. 249-264. ISSN: 1981-1179.

Recebido: 25.10 .2017

Aceito: 26.10 .2017

\section{Anexos}


1) Faixa etária:
( ) $18-22$
( ) $23-27$
( ) $28-32$
( ) $33-37$
( ) $38-42$
( ) 43 e mais

2) Estado civil:
( ) Solteiro (a)
( ) Casado (a)
( ) Viúvo (a)
( ) Outro:

3) Raça:
( ) Branco
( ) Negro
( ) Pardo
( ) Outro:

4) Escolaridade:
( ) Analfabeto
( ) Ensino fundamental incompleto
( ) Ensino fundamental completo
( ) Ensino médio incompleto
( ) Ensino médio completo
( ) Superior incompleto
( ) Superior completo

5) Renda:
( ) Sem renda / não sabe
( ) Até um salário mínimo
( ) De dois a três salários mínimos
( ) De quatro a seis salários mínimos
( ) Sete salários mínimos ou mais. 


\title{
Anexo B - Questionário WHOQOL - bref
}

\author{
Instrumento de Avaliação de Qualidade de Vida
}

\section{The World Health Organization Quality of Life - WHOQOL-bref}

\section{Instruções}

Este questionário é sobre como você se sente a respeito de sua qualidade de vida, saúde e outras áreas de sua vida. Por favor responda a todas as questões. Se você não tem certeza sobre que resposta dar em uma questão, por favor, escolha entre as alternativas a que lhe parece mais apropriada.

Esta, muitas vezes, poderá ser sua primeira escolha. Por favor, tenha em mente seus valores, aspirações, prazeres e preocupações. Nós estamos perguntando o que você acha de sua vida, tomando como como referência as duas últimas semanas. Por exemplo, pensando nas últimas duas semanas, uma questão poderia ser:

\begin{tabular}{|c|c|c|c|c|c|}
\hline & nada & $\begin{array}{c}\text { Muito } \\
\text { pouco }\end{array}$ & médio & muito & completamente \\
\hline $\begin{array}{c}\text { Você recebe dos outros o apoio de que } \\
\text { necessita? }\end{array}$ & 1 & 2 & 3 & 4 & 5 \\
\hline
\end{tabular}

Você deve circular o número que melhor corresponde ao quanto você recebe dos outros o apoio de que necessita nestas últimas duas semanas. Portanto, você deve circular o número 4 se você recebeu "muito" apoio como abaixo.

\begin{tabular}{|c|c|c|c|c|c|}
\hline & nada & $\begin{array}{c}\text { Muito } \\
\text { pouco }\end{array}$ & médio & muito & completamente \\
\hline $\begin{array}{c}\text { Você recebe dos outros o apoio de que } \\
\text { necessita? }\end{array}$ & 1 & 2 & 3 & (4) & 5 \\
\hline
\end{tabular}

Você deve circular o número 1 se você não recebeu "nada" de apoio. Por favor, leia cada questão, veja o que você acha e circule no número e lhe parece a melhor resposta.

\begin{tabular}{|c|c|c|c|c|c|c|}
\hline & & muito ruim & Ruim & nem ruim nem boa & boa & muito boa \\
\hline 1 & $\begin{array}{c}\text { Como você avaliaria sua } \\
\text { qualidade de vida? }\end{array}$ & 1 & 2 & 3 & 4 & 5 \\
\hline & $\begin{array}{c}\text { muito } \\
\text { insatisfeito }\end{array}$ & Insatisfeito & $\begin{array}{c}\text { nem satisfeito nem } \\
\text { insatisfeito }\end{array}$ & satisfeito & $\begin{array}{c}\text { muito } \\
\text { satisfeito }\end{array}$ \\
\hline 2 & $\begin{array}{c}\text { Quão satisfeito(a) você } \\
\text { está com a sua saúde? }\end{array}$ & 1 & 2 & 3 & 4 & 5 \\
\hline
\end{tabular}

As questões seguintes são sobre o quanto você tem sentido algumas coisas nas últimas duas semanas.

\begin{tabular}{|c|c|c|c|c|c|c|}
\hline & nada & $\begin{array}{c}\text { muito } \\
\text { pouco }\end{array}$ & $\begin{array}{c}\text { mais ou } \\
\text { menos }\end{array}$ & bastante & extremamente \\
\hline 3 & $\begin{array}{c}\text { Em que medida você acha que sua dor } \\
\text { (física) impede você de fazer o que você } \\
\text { precisa? }\end{array}$ & 1 & 2 & 3 & 4 & 5 \\
\hline 4 & $\begin{array}{c}\text { O quanto você precisa de algum } \\
\text { tratamento médico para levar sua vida } \\
\text { diária? }\end{array}$ & 1 & 2 & 3 & 4 & 5 \\
\hline 5 & O quanto você aproveita a vida? & 1 & 2 & 3 & 4 & 5 \\
\hline 6 & $\begin{array}{c}\text { Em que medida você acha que a sua vida } \\
\text { tem sentido? }\end{array}$ & 1 & 2 & 3 & 4 & 5 \\
\hline
\end{tabular}




\begin{tabular}{|c|c|c|c|c|c|c|}
\hline 7 & O quanto você consegue se concentrar? & 1 & 2 & 3 & 4 & 5 \\
\hline 8 & $\begin{array}{c}\text { Quão seguro(a) você se sente em sua vida } \\
\text { diária? }\end{array}$ & 1 & 2 & 3 & 4 & 5 \\
\hline 9 & $\begin{array}{c}\text { Quão saudável é o seu ambiente físico } \\
\text { (clima, barulho, poluição, atrativos)? }\end{array}$ & 1 & 2 & 3 & 4 & 5 \\
\hline
\end{tabular}

As questões seguintes perguntam sobre quão completamente você tem sentido ou é capaz de fazer certas coisas nestas últimas duas semanas.

\begin{tabular}{|c|c|c|c|c|c|c|}
\hline & nada & $\begin{array}{c}\text { muito } \\
\text { pouco }\end{array}$ & médio & muito & completamente \\
\hline 10 & $\begin{array}{c}\text { Você tem energia suficiente para seu dia-a- } \\
\text { dia? }\end{array}$ & 1 & 2 & 3 & 4 & 5 \\
\hline 11 & Você é capaz de aceitar sua aparência fisica? & 1 & 2 & 3 & 4 & 5 \\
\hline 12 & $\begin{array}{c}\text { Você tem dinheiro suficiente para satisfazer } \\
\text { suas necessidades? }\end{array}$ & 1 & 2 & 3 & 4 & 5 \\
\hline 13 & $\begin{array}{c}\text { Quão disponiveis para você estão as } \\
\text { informações que precisa no seu dia-a-dia? }\end{array}$ & 1 & 2 & 3 & 4 & 5 \\
\hline 14 & $\begin{array}{c}\text { Em que medida você tem oportunidades de } \\
\text { atividade de lazer? }\end{array}$ & 1 & 2 & 3 & 4 & 5 \\
\hline
\end{tabular}

As questões seguintes perguntam sobre quão bem ou satisfeito você se sentiu a respeito de vários aspectos de sua vida nas últimas duas semanas.

\begin{tabular}{|c|c|c|c|c|c|c|}
\hline & & muito ruim & ruim & $\begin{array}{l}\text { nem ruim } \\
\text { nem bom }\end{array}$ & bom & muito bom \\
\hline \multirow[t]{2}{*}{15} & $\begin{array}{l}\text { Quão bem você é capaz de } \\
\text { se locomover? }\end{array}$ & 1 & 2 & 3 & 4 & 5 \\
\hline & & $\begin{array}{c}\text { muito } \\
\text { insatisfeito }\end{array}$ & Insatisfeito & $\begin{array}{c}\text { nem satisfeito } \\
\text { nem } \\
\text { insatisfeito } \\
\end{array}$ & satisfeito & $\begin{array}{l}\text { Muito } \\
\text { satisfeito }\end{array}$ \\
\hline 16 & $\begin{array}{c}\text { Quão satisfeito(a) você está } \\
\text { com o seu sono? }\end{array}$ & 1 & 2 & 3 & 4 & 5 \\
\hline 17 & $\begin{array}{c}\text { Quão satisfeito(a) você está } \\
\text { com sua capacidade de } \\
\text { desempenhar as atividades } \\
\text { do seu dia-a-dia? }\end{array}$ & 1 & 2 & 3 & 4 & 5 \\
\hline 18 & $\begin{array}{l}\text { Quão satisfeito(a) você está } \\
\text { com sua capacidade para o } \\
\text { trabalho? }\end{array}$ & 1 & 2 & 3 & 4 & 5 \\
\hline 19 & $\begin{array}{c}\text { Quão satisfeito(a) você está } \\
\text { consigo mesmo? }\end{array}$ & 1 & 2 & 3 & 4 & 5 \\
\hline 20 & $\begin{array}{c}\text { Quão satisfeito(a) você está } \\
\text { com suas relações pessoais } \\
\text { (amigos, parentes, } \\
\text { conhecidos, colegas)? }\end{array}$ & 1 & 2 & 3 & 4 & 5 \\
\hline 21 & $\begin{array}{l}\text { Quão satisfeito(a) você está } \\
\text { com sua vida sexual? }\end{array}$ & 1 & 2 & 3 & 4 & 5 \\
\hline 22 & $\begin{array}{l}\text { Quão satisfeito(a) você está } \\
\text { com }\end{array}$ & 1 & 2 & 3 & 4 & 5 \\
\hline
\end{tabular}




\begin{tabular}{|c|c|c|c|c|c|c|}
\hline 23 & $\begin{array}{c}\text { seus amigos? } \\
\begin{array}{c}\text { Quão satisfeito(a) você está } \\
\text { com } \\
\text { as condições do local onde } \\
\text { mora? }\end{array}\end{array} 21 \quad \begin{array}{l}\text { Quão satisfeito(a) você está } \\
\text { com o } \\
\text { seu acesso aos serviços de } \\
\text { saúde? }\end{array}$ & 1 & 2 & 3 & 4 & 5 \\
\hline 25 & $\begin{array}{c}\text { Quão satisfeito(a) você está } \\
\text { com } \\
\text { o seu meio de transporte? }\end{array}$ & 1 & 2 & 3 & 4 & 5 \\
\hline
\end{tabular}

As questões seguintes referem-se a com que freqüência você sentiu ou experimentou certas coisas nas últimas duas semanas.

\begin{tabular}{|c|c|c|c|c|c|c|}
\hline 26 & nunca & $\begin{array}{c}\text { Algumas } \\
\text { vezes }\end{array}$ & freqüentemente & $\begin{array}{c}\text { muito } \\
\text { freqüentemente }\end{array}$ & sempre \\
\hline $\begin{array}{c}\text { Com que freqüência você tem } \\
\text { sentimentos negativos tais } \\
\text { como mau humor, desespero, } \\
\text { ansiedade, depressão? }\end{array}$ & 1 & 2 & 3 & 4 & 5 \\
\hline
\end{tabular}

Alguém lhe ajudou a preencher este questionário?

Quanto tempo você levou para preencher este questionário?

Você tem algum comentário sobre o questionário?

OBRIGADO PELA SUA COLABORAÇÃO

\section{Anexo C - Escala de Silhuetas}

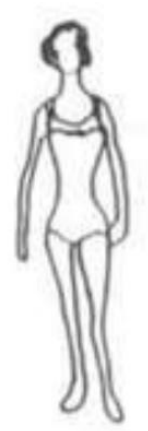

1

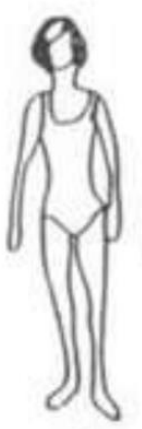

2

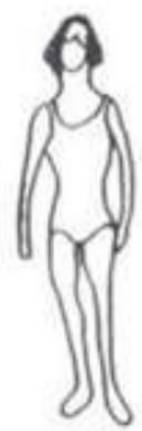

3

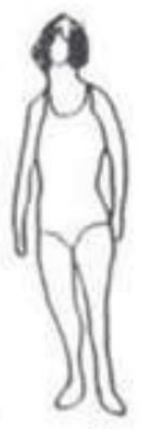

4

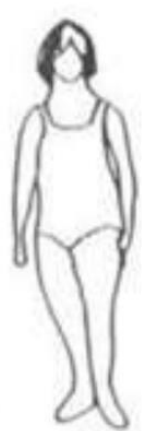

5

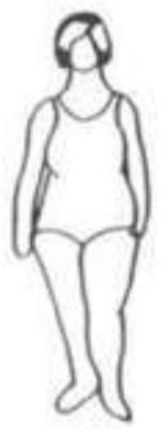

6

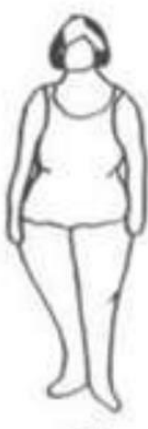

7

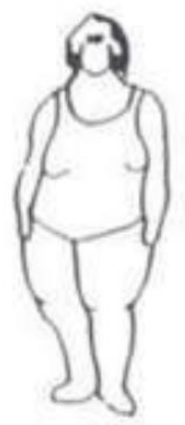

8

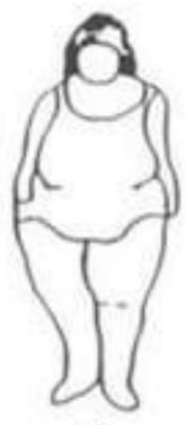

9

Escala de Silhuetas para adultos, onde A é feminino e B masculino. 